\title{
Simultaneously flippable edges in triangulations
}

\author{
Diane L. Souvaine ${ }^{1,3}$, Csaba D. Tóth ${ }^{2,4}$, Andrew Winslow ${ }^{1,3}$ \\ 1 Tufts University, Medford, MA, USA \\ dls@cs.tufts.edu, awinslow@cs.tufts.edu \\ ${ }^{2}$ University of Calgary, Calgary, AB, Canada \\ cdtoth@ucalgary.ca
}

\begin{abstract}
We show that every straight-line triangulation on $n$ vertices contains at least $(n-4) / 5$ simultaneously flippable edges. This bound is the best possible, and resolves an open problem by Galtier et al. .
\end{abstract}

Introduction. A (geometric) triangulation of a point set $P$ is a planar straight line graph with vertex set $P$ such that every bounded face is a triangle, and the outer face is the exterior of the convex hull of $P$. An edge $e$ of a triangulation is flippable if it is adjacent to two triangles, whose union is a convex quadrilateral $Q(e)$. A set $E$ of edges in a triangulation are simultaneously flippable if each edge in $E$ is flippable, and the quadrilaterals $Q(e), e \in E$, are pairwise interior disjoint.

For a triangulation $T_{P}$ of a point set $P$, let $f_{\text {sim }}(T)$ denote the the maximum number of simultaneously flippable edges in $T_{P}$, and let $f_{\operatorname{sim}}(n)=\min _{T_{P}:|P|=n} f_{\operatorname{sim}}\left(T_{P}\right)$ be the minimum of $f_{\text {sim }}(T)$ over all $n$-element point sets in general position in the plane. The value of $f_{\operatorname{sim}}(n)$ played a key role in recent results on the number of various classes of planar straight line graph embedded on given point sets $[\mathbf{2}, \mathbf{4}]$. Hurtado et al. [5] proved that every triangulation on $n$ vertices admits at least $(n-4) / 2$ flippable edges, and this bound cannot be improved in general. Galtier et al. $[\mathbf{3}]$ proved that $f_{\operatorname{sim}}(n) \geq(n-4) / 6$, and that there are triangulations $T_{P}$ with $|P|=n$ such that $f_{\operatorname{sim}}\left(T_{P}\right) \leq(n-4) / 5$, which is the best possible. In this note we improve the lower bound to $f_{\operatorname{sim}}(n) \geq(n-4) / 5$. This resolves an open problem posed in $[\mathbf{3}]$ and restated in $[\mathbf{1}]$.

LOWER BOUnd. Fix a set $P$ of $n$ points in general position in the plane, $h$ of which lie on the boundary of the convex hull, and fix a triangulation $T=T_{P}$. Then $T$ has exactly $3 n-h-3$ edges and $2 n-h-2$ bounded faces.

Separable edges. Following the terminology in [4], we say that an edge $e=u v$ of the triangulation is separable at vertex $u$ iff there is a line $\ell_{u}$ through $u$ such that $u v$ is the only edge incident to $u$ on one side of $\ell_{u}$. We use the following observations from [5]: An edge $u v$ of $T$ is flippable iff it is separable at neither endpoint. If $u$ is a hull vertex, then only the two incident hull edges are separable at $u$. Suppose now that $u$ is a vertex in the interior of the convex hull. If $u$ has degree 3 , then all three incident edges are separable at $u$. If $u$ has degree 4 or higher, then at most two edges are separable at $u$ and these edges must be consecutive in the rotation of $u$.

Similarly to [5], we assign every non-flippable edge $e$ to an incident vertex at which it is separable. If $e$ lies on the boundary of the convex hull, assign $e$ to its counterclockwise first hull vertex. If $e$ is incident to an interior vertex of degree 3, then assign $e$ to this

\footnotetext{
${ }^{3}$ Research supported in part by NSF grants CCF-0830734 and CBET-0941538.

${ }^{4}$ Research supported in part by NSERC grant RGPIN 35586.
} 
vertex. Otherwise assign $e$ to one of its endpoints at which it is separable (breaking ties arbitrarily).

Based on the above observations, we can now distinguish five types of vertices. Let $h$ be the number of hull vertices (with $h \geq 3$ ) and let $n_{3}$ be the number of interior vertices of degree 3 . Denote by $n_{4,0}, n_{4,1}$, and $n_{4,2}$ the number of interior vertices of degree 4 or higher which 0,1 , and 2 non-flippable edges, respectively are assigned. We have

$$
n=h+n_{4,2}+n_{4,1}+n_{4,0}+n_{3} .
$$

Using this notation, the number of non-flippable edges is exactly $h+3 n_{3}+2 n_{4,2}+n_{4,1}$. Denoting by $f$ the total number of flippable edges in $T$, we use (1) to get $f=(3 n-h-$ $3)-\left(h+3 n_{3}+2 n_{4,2}+n_{4,1}\right)$, or

$$
f=h+n_{4,2}+2 n_{4,1}+3 n_{4,0}-3 .
$$

Coloring argument. Galtier et al. $[\mathbf{3}]$ proved that every set of flippable edges in a triangulation is 3 -colorable such that each color class is simultaneously flippable. This implies, in particular, that every set of $k$ flippable edges in a triangulation contains a subset of at least $k / 3$ simultaneously flippable edges. This result, combined with a lower bound of $(n-4) / 2$ on the total number of flippable edges immediately gives $f_{\text {sim }} \geq(n-4) / 6$. We improve this lower bound to $f_{\text {sim }}(n) \geq(n-4) / 5$.

If $f \geq 3\lceil(n-4) / 5\rceil-2$, then the above 3 -coloring argument implies that the largest color classes contains at least $\lceil(n-4) / 5\rceil$ simultaneously flippable edges, as required. In the remainder of the proof, we assume that

$$
f \leq 3\left\lceil\frac{n-4}{5}\right\rceil-3 \leq \frac{3 n}{5}-3 .
$$

Recall that we have $f \geq \frac{1}{2}(n-4)$ by the result of Hurtado et al. [5]. So the number of flippable edges must be in the range $0.5 n-2 \leq f<0.6 n-3$. Combining (2) and (3), we have

$$
\frac{3}{5} n \geq h+n_{4,2}+2 n_{4,1}+3 n_{4,0} .
$$

We apply the 3-coloring result by Galtier et al. [3] only for a subset of the flippable edges. We call a flippable edge $e$ isolated if the convex quadrilateral $Q(e)$ is bounded by 4 non-flippable edges. It is clear that an isolated flippable edge is simultaneously flippable with any other flippable edge. Let $f_{0}$ and $f_{1}$ denote the number of isolated and nonisolated flippable edges, respectively, with $f=f_{0}+f_{1}$. Applying the 3 -coloring argument for the non-isolated flippable edges only, the number of simultaneously flippable edges is bounded by

$$
f_{\text {sim }} \geq f_{0}+\frac{f_{1}}{3}=\left(f-f_{1}\right)+\frac{f_{1}}{3}=f-\frac{2}{3} f_{1} .
$$

An auxiliary triangulation. Similarly to Hurtado et al. [5] and Hoffmann et al. [4], we use an auxiliary triangulation $\widehat{T}$. We construct $\widehat{T}$ from $T$ as follows:

(1) Add an auxiliary vertex $w$ in the exterior of the convex hull, and connect it to all hull vertices.

(2) Remove all interior vertices of degree 3 (and all incident edges).

Notice that only nonflippable edges have been deleted from $T$. In the triangulation $\widehat{T}$, the number of vertices is $n-n_{3}+1=h+n_{4,0}+n_{4,1}+n_{4,2}+1$ and all faces (including 
the unbounded face) are triangles. By Euler's formula, the number of faces in $\widehat{T}$ is:

$$
m=2\left(n-n_{3}+1\right)-4=2 h+2 n_{4,2}+2 n_{4,1}+2 n_{4,0}-2 .
$$

We 2-color the faces of $\widehat{T}$ as follows: let all triangles incident to vertex $w$ be white; let all triangles obtained by deleting a vertex of degree 3 be white; for each of the $n_{4,2}$ vertices (which have degree 4 or higher in $T$ and two assigned consecutive separable edges), let the triangle adjacent to both nonflippable edges be white; finally, color all remaining triangles of $\widehat{T}$ gray. See Figure 1 for an example.

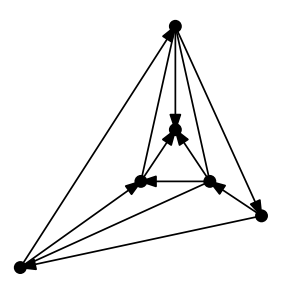

$T$

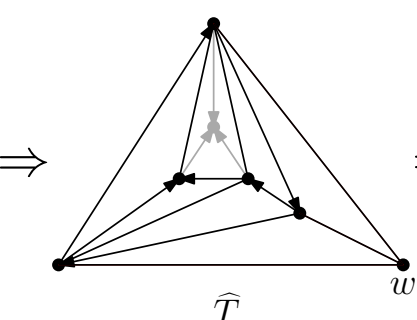

$\widehat{T}$

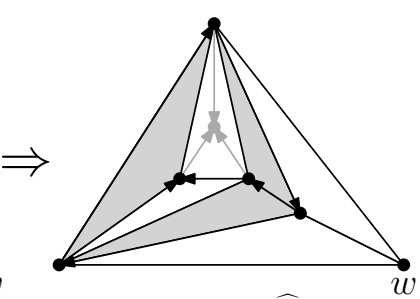

2-colored $\widehat{T}$

FIgURE 1. The 2-colored auxiliary triangulation $\widehat{T}$ of a triangulation $T$.

Under this coloring, the number of white faces is $m_{\text {white }}=h+n_{4,2}+n_{3}$. Using (6), the number of gray faces is

$$
\begin{aligned}
m_{\text {gray }} & =m-m_{\text {white }} \\
& =\left(2 h+2 n_{4,2}+2 n_{4,1}+2 n_{4,0}-2\right)-\left(h+n_{4,2}+n_{3}\right) \\
& =h+n_{4,2}+2 n_{4,1}+2 n_{4,0}-n_{3}-2 .
\end{aligned}
$$

Putting it all together. Observe that if a flippable edge $e$ of $T$ lies on the common boundary of two white triangles in the auxiliary graph $\widehat{T}$, then $e$ is isolated. That is, if $e$ is a nonisolated flippable edge in $T$, then it is on the boundary of a gray triangle in $\widehat{T}$. Since every gray triangle has three edges, the number of nonisolated flippable edges in $T$ is at most $3 m_{\text {gray }}$. Substituting this into our bound $(7)$ on the number of simultaneously flippable edges, we have

$$
\begin{aligned}
f_{\text {sim }} & \geq f-\frac{2}{3} f_{1} \\
& \geq f-2 m_{\text {gray }} \\
& =\left(h+n_{4,2}+2 n_{4,1}+3 n_{4,0}-3\right)-2\left(h+n_{4,2}+2 n_{4,1}+2 n_{4,0}-2-n_{3}\right) \\
& =2 n_{3}-h-n_{4,2}-2 n_{4,1}-n_{4,0}+1 .
\end{aligned}
$$

Finally, combining twice (1) minus three times (4), we obtain

$$
\begin{aligned}
2 n-3 \cdot \frac{3 n}{5} & \leq 2\left(h+n_{4,2}+n_{4,1}+n_{4,0}+n_{3}\right)-3\left(h+n_{4,2}+2 n_{4,1}+3 n_{4,0}\right) \\
\frac{n}{5} & \leq 2 n_{3}-h-n_{4,2}-4 n_{4,1}-7 n_{4,0} \\
& <2 n_{3}-h-n_{4,2}-2 n_{4,1}-n_{4,0}+1 \\
& \leq f_{\text {sim. }} .
\end{aligned}
$$

Under the condition (3), we have proved a lower bound of $f_{\text {sim }}>n / 5$; otherwise we have $f_{\text {sim }} \geq(n-4) / 5$, as required. 
UPPER BOUND CONSTRUCTIONS. In this section we construct an infinite family of geometric triangulations with at most $(n-4) / 5$ simultaneously flippable edges. This family includes all triangulations constructed by Galtier et al. [3]. First observe that a straight line drawing of $K_{4}$ has no flippable edge. We introduce two operations that each increase the number of vertices by 5 , and the maximum number of simultaneously flippable edges by one.
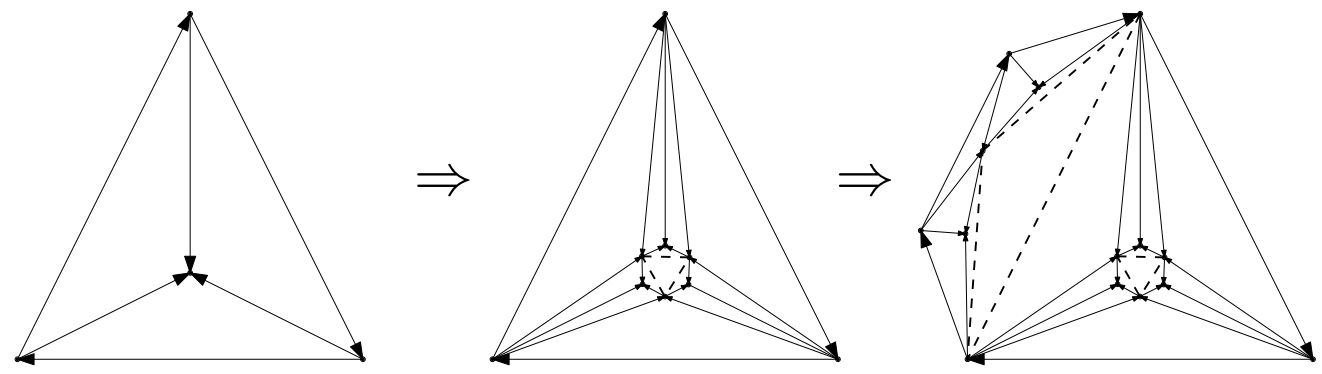

FIGURE 2. The two operations applied successively to $K_{4}$.

One operation replaces an interior vertex of degree 3 by a configuration of 6 vertices as shown at left in Fig. 2. The other operation adds 5 vertices in a close neighborhood of a hull edge as shown at right in Fig. 2. Note that both operations maintain the property that the triangles adjacent to the convex hull have no flippable edges. Each operation creates three new flippable edges, which form a triangle, so no two of them are simultaneously flippable. Each operation increases $h+n_{4,2}$ by 3 and $n_{3}$ by 2 , as expected based on the previous section.

Let $\mathcal{F}_{\text {sim }}$ denote the family of all geometric triangulations obtained from $K_{4}$ via applying an arbitrary sequence of the two operations. Then every triangulation $T \in \mathcal{F}_{\text {sim }}$ on $n$ vertices has at most $(n-4) / 5$ simultaneously flippable edges, attaining our lower bound for $f_{\operatorname{sim}}(n)$. We note that all upper bound constructions by Galtier et al. [3] can be obtained by applying our 2nd operation successively to all sides, starting from $K_{4}$.

\section{References}

[1] P. Bose and F. Hurtado, Flips in planar graphs, Comput. Geom. Theory Appl. 42 (1) (2009), 60-80.

[2] A. Dumitrescu, A. Schulz, A. Sheffer, and Cs. D. Tóth, Bounds on the maximum multiplicity of some common geometric graphs, in Proc. STACS, LIPICS, 2011, to appear.

[3] J. Galtier, F. Hurtado, M. Noy, S. Pérennes, and J. Urrutia, Simultaneous edge flipping in triangulations, Internat. J. Comput. Geom. Appl. 13 (2) (2003), 113-133.

[4] M. Hoffmann, M. Sharir, A. Sheffer, Cs. D. Tóth, and Emo Welzl, Counting plane graphs: Flippability and its applications, submitted manuscript, 2011. http://arxiv.org/abs/1012.0591.

[5] F. Hurtado, M. Noy, and J. Urrutia, Flipping edges in triangulations, Discrete Comput. Geom. 22 (1999), 333-346.

[6] J. Urrutia, Flipping edges in triangulations of point sets, polygons and maximal planar graphs, invited talk at the 1st Japan Conf. on Discrete and Comput. Geom. (Tokyo, November 17-20, 1997). http://www.matem. unam.mx/ urrutia/online_papers/TrianSurv.pdf 